\title{
Preface
}

\section{Managing Your Anesthesiology Practice for the Future}

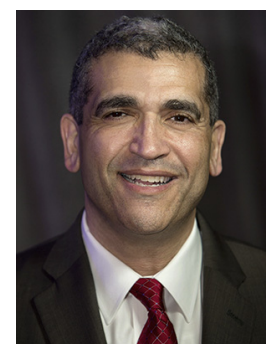

Amr E. Abouleish, MD, MBA, FASA Stanley W. Stead, MD, MBA, FASA

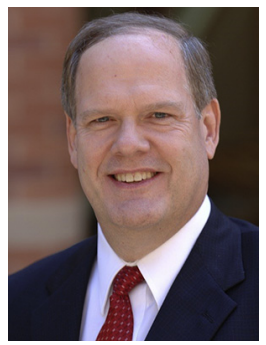
Editors

.

Managing your anesthesiology practice for the future continues to be a challenge. In the past, anesthesiology practices focused on volume: maximizing unit production, the conversion factor rate, and account collections. Successful practices minimized their overall costs of billing and administration and carefully managed the number of anesthetizing locations. But health care delivery systems are evolving, and successful anesthesiology groups must expand their traditional focus to meet new demands and challenges. In this issue of Anesthesiology Clinics, we present thirteen articles that focus on these new demands and challenges. First, you must be able to measure your production, costs, and quality. Second, anesthesiology practices must expand their comfort zone of the operating room (OR) to include the perioperative period for presurgical optimization, multimodal pain management, and postoperative recovery as exemplified by the perioperative surgical home (PSH). Finally, the traditional divisions of academic and private practice have become blurred to encompass the skills and capabilities of both divisions moving forward.

\section{OPERATING ROOM MANAGEMENT}

Even as anesthesiology practices are asked to expand their duties, the primary challenge of surgical anesthesia continues. But unlike previous years, many anesthesiology groups do not simply cover one facility but now are part of large groups or national companies that cover multiple facilities. With this expansion of coverage, groups are trying to make data-driven evaluations on productivity and OR efficiencies. Hudson and Lebovitz present their lessons learned from comparing clinical productivity among 14 different facilities. Morris and colleagues describe principles of OR efficiency and how they apply to a real-world scenario: one surgeon wanting to "flip" 
cases in two rooms. Methods and measures are described for monitoring overlapping surgery, auditing, providing accountability and metrics of surgical production.

\section{QUALITY AND REPORTING}

Quality outcome reporting has become an important requirement of many practices, not only to federal payers but also to the practice, facilities, and other payers. Focusing on individual clinicians, Allyn and Curry describe a robust quality system based upon the data collected from their department that has evolved over 20 years. They describe how to capture data, how to provide meaningful reporting of the information, and how to use that data to demonstrate the value their practice brings to their institution. Tung reminds the reader of the challenges of outcome reporting: avoiding direct comparisons between clinicians, while still providing a framework for institutional improvement of outcome reporting. In contrast to Allyn and Curry, Popovich and Schmitz focus on group quality reporting. They discuss measure development and how those measures are captured into clinical data registries. They provide readers with a wealth of information on reporting and how to select a registry for reporting quality outcome measures. Facilities are also very interested in developing quality organizations. Rosinia and colleagues expand the concepts of quality reporting and safety into principles for anesthesiology's development of a high-reliability organization in intensive care and ORs.

\section{ANESTHESIOLOGY'S VALUE PROPOSITION}

Anesthesiology practices must continue to evolve and articulate measurable and demonstrable benefits that patients, facilities, other providers, and payers receive from an anesthesia service. Practices must be able to compete by moving from piecework to bundled payments. Those bundles must encompass the continuum of care from the decision to operate to recovery of function. Termed the PSH, practices must optimize the patient for surgery, manage their recovery and pain management, and encompass population health. Szokol and Chamberlin analyze the strengths, weaknesses, and opportunities practices face. They conclude with recommendations that practice must seize: leadership, patient satisfaction, and incorporation of the PSH into their practice. Stead and Merrick take readers through the considerations and analysis of the services and costs of providing anesthesia services for an episode of care. They show the calculations and decisions necessary to develop a bundled payment for all anesthesia services needed during an episode of surgical care.

Anesthesiology's expanded role in preoperative assessment and preparation for surgery is highlighted by Shah and Vetter. They articulate the use of graded assessments from mobile technologies to face-to-face visits to assess comorbidities, optimize patients, and provide prehabilitation with targeted therapeutic interventions. Principles of PSH can also be applied to pain medicine. Pozek and colleagues illustrate how improved and coordinated acute pain management of surgical patients can have even more impact than simply shortened length of stay. Careful preoperative assessment of risk factors, including behavioral risk factors, perioperative management with multimodal nonopioid analgesia, and regional and neuraxial techniques, can minimize postsurgical pain and minimize the risk of chronic postsurgical pain. Khan and Manion describe how the PSH can provide an optimal framework for complex chronic pain patients. Chronic pain patients benefit from preoperative assessment with optimization in planning for surgery, with the use of intraoperative 
multimodal analgesia. Postoperative and postdischarge care coordination care pathways in the PSH are essential for chronic pain patients.

Schweitzer believes that expansion beyond the PSH to engaging anesthesiologists and the surgical specialists in population health will improve quality, reduce costs, and provide more effective care management processes. He encourages anesthesiologists to participate in accountable care organizations and population health management organizations to develop "shadow bundles" to improve quality and reduce costs.

In the final article, Regev and Mahajan describe the opportunities and challenges in merging academic and private practice anesthesiology groups. These mergers are often done to gain efficiencies and synergies, but the differences between these two types of practices in their workforces and workflow can affect the overall success of a merger.

Amr E. Abouleish, MD, MBA, FASA

Department of Anesthesiology The University of Texas Medical Branch

301 University Boulevard Galveston, TX 77555-0877, USA

Stanley W. Stead, MD, MBA, FASA

Stead Health Group, Inc 4819 Andasol Avenue, Suite 100 Encino, CA 91316-3802, USA

E-mail addresses:

aaboulei@utmb.edu (A.E. Abouleish) sstead@gmail.com (S.W. Stead) 